\title{
Efficient CsPbBr3 Perovskite Light-Emitting Diodes Enabled by Synergetic Morphology Control
}

Li-Peng Cheng, Jing-Sheng Huang, Yang Shen, Guo-Peng Li, Xiaoke Liu, Wei Li, YuHan Wang, Yan-Qing Li, Yang Jiang, Feng Gao, Chun-Sing Lee and Jian-Xin Tang

The self-archived postprint version of this journal article is available at Linköping University Institutional Repository (DiVA):

http://urn.kb.se/resolve?urn=urn:nbn:se:liu:diva-15.5000

N.B.: When citing this work, cite the original publication.

Cheng, L., Huang, J., Shen, Y., Li, G., Liu, X., Li, W., Wang, Y., Li, Y., Jiang, Y., Gao, F., Lee, C., Tang, J., (2019), Efficient CsPbBr3 Perovskite Light-Emitting Diodes Enabled by Synergetic Morphology Control, Advanced Optical Materials, 7(4), 1801534. https://doi.org/10.1002/adom.201801534

Original publication available at:

https://doi.org/10.1002/adom.201801534

Copyright: WILEY-V C H VERLAG GMBH

Publisher URL Missing 


\section{WILEY-VCH}

DOI: $10.1002 /(($ please add manuscript number $))$

Article type: Communication

\section{Efficient CsPbBr3 Perovskite Light-Emitting Diodes Enabled by Synergetic Morphology Control}

Li-Peng Cheng, Jing-Sheng Huang, Yang Shen, Guo-Peng Li, Xiao-Ke Liu, Wei Li, Yu-Han Wang, Yan-Qing Li, * Yang Jiang, Feng Gao, * Chun-Sing Lee, * and Jian-Xin Tang*

L. P. Cheng, J. S. Huang, Y. Shen, W. Li, Y. H. Wang, Prof. Y. Q. Li, Prof. J. X. Tang Institute of Functional Nano \& Soft Materials (FUNSOM), Jiangsu Key Laboratory for Carbon-Based Functional Materials \& Devices, Soochow University, Suzhou 215123, China E-mail: jxtang@suda.edu.cn (J.X. Tang),yqli@suda.edu.cn (Y.Q. Li)

Dr. G. P. Li, Prof. Y. Jiang

School of Materials Science and Engineering, Hefei University of Technology (HFUT), Hefei, Anhui, 230009, P. R. China

Dr. X. K. Liu, Prof. F. Gao

Biomolecular and Organic Electronics, IFM, Linköping University, 58183 Linköping, Sweden

E-mail: fenga@ifm.liu.se (F. Gao)

Prof. C.-S. Lee

Center of Super-Diamond and Advanced Film (COSADF) and Department of Chemistry, City University of Hong Kong, Hong Kong S.A.R., P.R. China

E-mail: apcslee@cityu.edu.hk

Prof. J. X. Tang

Institute of Organic Optoelectronics (IOO), JITRI, Wujiang, Suzhou, China

Keywords: $\mathrm{CsPbBr}_{3}$, all-inorganic perovskite film, perovskite light-emitting diodes, morphology control

The development of solution-processed all-inorganic perovskite light-emitting diodes (PeLEDs) is currently hindered by low emission efficiency due to morphological defects and severe non-radiative recombination in all-inorganic perovskite emitter. Herein, bright PeLEDs are demonstrated by synergetic morphology control over cesium lead bromide $\left(\mathrm{Cs}_{\mathrm{PbBr}}\right)$ perovskite films with the combination of two additives. The phenethylammonium bromide ( $\mathrm{PEABr}$ ) additive enables the formation of mixed-dimensional $\mathrm{Cs} \mathrm{PbBr}_{3}$ perovskites featuring 


\section{WILEY-VCH}

the reduced grain size $(<15 \mathrm{~nm})$ and efficient energy funneling, while the dielectric polyethyleneglycol (PEG) additive promotes the formation of highly compact and pinholefree perovskite films with defect passivation at grain boundaries. Consequently, all-inorganic green PeLEDs achieve a current efficiency of $37.14 \mathrm{~cd} \mathrm{~A}^{-1}$ and an external quantum efficiency of $13.14 \%$ with the maximum brightness up to $45990 \mathrm{~cd} \mathrm{~m}^{-2}$ and high color purity. Furthermore, this method can be effectively extended to realize flexible PeLEDs on plastic substrates with a high efficiency of $31.0 \mathrm{~cd} \mathrm{~A}^{-1}$.

\section{Introduction}

Solution-processed metal halide perovskite light-emitting diodes (PeLEDs) are attracting great attention for the applications in next-generation optoelectronics due to their high photoluminescence quantum-yield (PLQY), easily tunable bandgap, high color purity, and excellent charge transport properties. ${ }^{[1-10]}$ Since the first report on PeLEDs using organicinorganic hybrid perovskites (e.g., $\mathrm{CH}_{3} \mathrm{NH}_{3} \mathrm{PbBr}_{3}$ ) as the emitter, ${ }^{[1]}$ their external quantum efficiency (EQE) has risen dramatically from initial $0.76 \%$ to $14.36 \%$ for red and green emissions. ${ }^{[11]}$ Regardless of the rapid progress in PeLEDs, ${ }^{[1,6,11-13]}$ chemical and thermal stabilities of organic-inorganic hybrid perovskites are subject to a debate due to the weak ion binding force between organic cation and metal anion. As a comparison, all-inorganic perovskites like cesium lead bromide $\left(\mathrm{CsPbr}_{3}\right)$ hold great promise as an alternative emitter to circumvent the issue on stability. ${ }^{[4,7-10,14-18]}$

However, device performance of all-inorganic PeLEDs is commonly limited with low brightness and EQE due to the severe current leakage and high non-radiative recombination losses, which are caused by incomplete surface coverage and increased defects at grain boundaries. In the past few years, various approaches have been proposed to enhance the device performance of $\mathrm{CsPbr}_{3}$-based $\mathrm{PeLEDs}$ through morphology control over $\mathrm{CsPbBr}_{3}$ perovskite films or the incorporation of $\mathrm{CsPbBr}_{3}$ quantum-dots. ${ }^{[7-9,14-18]}$ For example, CsBr- 


\section{WILEY-VCH}

rich precursor solution was usually prepared to reduce defect density without changing the crystal structure of the $\mathrm{CsPbBr}_{3}$ perovskites. ${ }^{[18]}$ Additives were also introduced into perovskite precursor solutions to improve film uniformity and coverage with small grains that can spatially confine exciton diffusion or reduce defect density at the grain boundaries to suppress the photoluminescence (PL) quenching. ${ }^{[6,14]}$ Recently, an efficient $\mathrm{CsPbBr}_{3}$ PeLED has been demonstrated with a peak EQE of $10.4 \%$, in which an organic cation methylammonium bromide $(\mathrm{MABr})$ was incorporated into the $\mathrm{CsPbr}_{3}$ perovskite. ${ }^{[19]}$

Despite of the progress abovementioned, the $\mathrm{CsPbBr}_{3}$ perovskite with bulk threedimensional (3D) crystal structure has small exciton binding energy ( $\sim 40 \mathrm{meV})$ and long charge diffusion length, ${ }^{[2]}$ giving rise to low PLQYs at low excitation densities due to the nature of carrier density dependence of bimolecular recombination. To overcome this limitation, an effective strategy is to reduce the grain size in perovskite films to spatially confine the charge carriers for the enhancement of radiative monomolecular (excitonic) recombination. ${ }^{[1,6]}$ In addition, two-dimensional (2D) Ruddlesden-Popper perovskites with layered structures have been developed recently by intercalating large organic counter-cations within the lead halide networks, leading to higher PL efficiency due to enlarged exciton binding energy and concentrated charge carriers. ${ }^{[1,12,20-23]}$ For instance, phenethylammonium bromide ( $\mathrm{PEABr}$ ) has been incorporated to form mixed-dimensional $\mathrm{CsPbBr}_{3}$ perovskites, which can facilitate the radiative recombination along with higher PLQYs and improved device performance in PeLEDs due to efficient energy funneling from larger bandgap (2D) domains to the lowest bandgap (3D) radiative domains. ${ }^{[11,12,20,21]}$ To further optimize the allinorganic PeLEDs, it is of critical importance to explore an effective strategy for fabricating smooth and pinhole-free $\mathrm{CsPbBr}_{3}$ perovskite films with improved morphological and photophysical properties.

Here, we demonstrate synergetic morphology control over $\mathrm{CsPbBr} 3$ perovskite films by incorporating two additives, PEABr and polyethyleneglycol (PEG), to achieve all-inorganic 


\section{WILEY-VCH}

PeLEDs with high brightness and EQEs. PEABr enables the formation of mixed-dimensional

$\mathrm{CsPBr}_{3}$ perovskites featuring reduced grain size and efficient energy funneling, while PEG promotes the uniformity of perovskite films with higher surface coverage and reduced defect density at grain boundaries. Smooth and pinhole-free $\mathrm{CsPbBr}_{3}$ perovskite films with suppressed current leakage and non-radiative recombination losses are obtained, allowing a substantial increase in both brightness and efficiency of all-inorganic PeLEDs with green emission. The best PeLED yields a current efficiency (CE) of $37.14 \mathrm{~cd} \mathrm{~A}^{-1}$, an EQE of $13.14 \%$, a maximum luminance of $45,990 \mathrm{~cd} \mathrm{~m}^{-2}$ and high color purity with the full-width at half-maximum (FWHM) of $18 \mathrm{~nm}$. Furthermore, efficient flexible PeLEDs with state-of-theart performance are realized on plastic substrates, showing a $\mathrm{CE}$ of $31.0 \mathrm{~cd} \mathrm{~A}^{-1}$ and an EQE of $10.1 \%$.

\section{Results and Discussion}

\subsection{CsPbBr3 Perovskite Films}

Figure 1a presents schematic synthetic procedures of the $\mathrm{CsPbBr}$ perovskite films with the PEG and PEABr additives (see the details in Experimental Section). The transparent $\mathrm{CsPbBr}_{3}$ precursor solutions were prepared by mixing the solution of cesium bromide $(\mathrm{CsBr})$, lead bromide $\left(\mathrm{PbBr}_{2}\right)$ and PEABr in dimethyl sulfoxide (DMSO) with the PEG solution in different weight ratios (see their chemical structures in Figure 1b). Here, excess CsBr was used in the $\mathrm{CsPBr}_{3}$ precursor solution to minimize the formation of metallic $\mathrm{Pb}$ atoms and thereby suppress the exciton quenching. ${ }^{[18]}$ The molar ratio of $\mathrm{CsBr}: \mathrm{PbBr}_{2}$ was optimized to be $1.5: 1$ in the non-stoichiometric solution which delivers the best device performance in PeLEDs (Table S1). The $\mathrm{CsPbBr}_{3}$ perovskite films were obtained by spin-coating the precursor solution on the indium-tin-oxide (ITO) glass substrates coated with poly $(3,4-$ ethylenedioxythiophene):poly(styrenesulfonate) (PEDOT:PSS), followed by thermal annealing. 


\section{WILEY-VCH}

Figure 2a displays X-ray diffraction (XRD) patterns of $\mathrm{CsPbBr}_{3}$ perovskite films with/without additive(s). These $\mathrm{CsPbBr}_{3}$ perovskite films are dominated by an orthorhombic (Pnma) crystal structure, consistent with the previous reports. ${ }^{[14,18,24]}$ The characteristic peaks at $15.1^{\circ}, 21.5^{\circ}, 30.3^{\circ}, 35.3^{\circ}$, and $37.6^{\circ}$ can be assigned to (101), (121), (202), (141), and (321) crystal planes, respectively. Moreover, the $\mathrm{CsPbBr}_{3}$ perovskite films maintain the identical XRD patterns with the variation of PEABr or PEG content, indicating that the addition of a small amount of PEABr or PEG additive does not significantly affect the intrinsic crystal structure (see Figure S1a). ${ }^{[14,20]}$

The morphology of the $\mathrm{Cs} \mathrm{PbBr}_{3}$ perovskite films on PEDOT:PSS-coated substrates was characterized by scanning electron microscopy (SEM). As shown in Figure 2b, pure $\mathrm{CsPbBr}_{3}$ film without any additive shows incomplete surface coverage with discrete large grains $(\sim 200$ $\mathrm{nm}$ ), probably leading to severe current leakage and limiting the device performance of PeLEDs. As a comparison, the morphology of the $\mathrm{CsPbBr}_{3}$ perovskite films can be dramatically improved with the addition of PEABr or/and PEG. It is evident in Figure $2 \mathrm{~b}$ that the $\mathrm{PEABr}$ additive enables the growth of small grains in the $\mathrm{Cs} \mathrm{PbBr}_{3}$ perovskite films (hereafter termed $\mathrm{CsPbBr}_{3}+\mathrm{PEABr}$ ), whereas the $\mathrm{CsPbBr}_{3}$ film with $\mathrm{PEG}$ (hereafter termed $\mathrm{CsPbBr}_{3}+\mathrm{PEG}$ ) exhibits compact morphology with high surface coverage and few pinholes. More importantly, PEABr and PEG have synergetic effects on the morphology control over $\mathrm{CsPbBr}_{3}$ perovskites (hereafter termed $\mathrm{CsPbBr}_{3}+\mathrm{PEG}+\mathrm{PEABr}$ ), leading to smooth and pinhole-free films with small grains $(<15 \mathrm{~nm})$. However, it is worth noting that the weight ratios of $\mathrm{PEABr}$ and $\mathrm{PEG}$ to $\mathrm{CsPbr}_{3}$ in the premixed precursor solutions are crucial for the perovskite film growth (Figure S1). For example, a large amount of $\operatorname{PEABr}(>20 \%)$ will result in rough surface of the $\mathrm{CsPbBr}_{3}$ perovskite films owing to the increased hydrophobicity (Figure S1b). The optimal weight ratios of $\mathrm{PEABr}$ and $\mathrm{PEG}$ to $\mathrm{CsPbBr}_{3}$ are about $10 \%$ and $3.8 \%$, respectively, by taking into account the effects of PEABr and PEG contents on film morphology, optical properties and device performance (Figures S1-S5). Under such optimal 


\section{WILEY-VCH}

conditions, the $\mathrm{Cs} \mathrm{PbBr}_{3}+\mathrm{PEG}+\mathrm{PEABr}$ perovskite films exhibit a smooth morphology with the highest surface coverage and the most uniform grain sizes $(<15 \mathrm{~nm})$.

Figure 2c displays the optical properties of various $\mathrm{CsPBr}_{3}$ perovskite films on PEDOT:PSS. The typical absorption peak of pure $\mathrm{CsPbBr}_{3}$ appears at around $520 \mathrm{~nm}$ (direct bandgap $\approx 2.38 \mathrm{eV}$ ), while the corresponding PL peak remains at the same position, indicating a low exciton binding energy in the $\mathrm{CsPBr}_{3}$ perovskite. ${ }^{[20]}$ There is no obvious change in the absorption and PL peak positions in the $\mathrm{CsPbBr}_{3}+\mathrm{PEG}$ perovskite film (Figure 2c), while its PL intensity is strongly enhanced under the same ultraviolet (UV) illumination (see the photo in Figure 2c). Compared to the $\mathrm{CsPbBr}_{3}+\mathrm{PEG}$ perovskite film, the addition of $\mathrm{PEABr}$ induces blue-shifts of the absorption and $\mathrm{PL}$ peaks in either $\mathrm{CsPbBr}_{3}+\mathrm{PEABr}$ or $\mathrm{CsPbr}_{3}+\mathrm{PEG}+\mathrm{PEABr}$ perovskite film (Figure 2c). It is evident that increasing $\mathrm{PEABr}$ content induces the gradual blue-shift of the absorption and PL peaks in the $\mathrm{CsPbBr}_{3}+\mathrm{PEG}+\mathrm{PEABr}$ perovskite film (Figure S3). More interestingly, when the weight ratio of the PEABr additive in the precursor solution is or exceeds $10 \%$, four additional excitonic absorption peaks can be observed at the short wavelength region and become more apparent along with the attenuation of the bandgap absorption peak at about $510 \mathrm{~nm}$ (Figure S3a). According to previous reports, ${ }^{[20,22,24,25]}$ such a phenomenon originates from the formation of layered perovskite structures in the formula of $\mathrm{PEA}_{2}\left(\mathrm{CsPbBr}_{3}\right)_{n-1} \mathrm{PbBr}_{4}$, where $n$ is the number of the $\mathrm{PbBr}_{4}{ }^{2-}$ octahedra sheets between two organic PEA insulating layers. Accordingly, $n=$ 1 and $n=\infty$ correspond to pure 2D (PEA) $)_{2} \mathrm{PbBr}_{4}$ phase and bulk $3 \mathrm{D} \mathrm{CsPbBr}_{3}$ phase, respectively. With smaller $n$ numbers, quantum confinement effect will be dominant with the increase in the bandgap and exciton energies. ${ }^{[20]}$ Therefore, those four excitonic absorption peaks centered at about $400 \mathrm{~nm}, 430 \mathrm{~nm}, 460 \mathrm{~nm}$ and $480 \mathrm{~nm}$ (Figure S3a) can be assigned to quasi-2D $\mathrm{PEA}_{2}\left(\mathrm{CsPbBr}_{3}\right)_{n-1} \mathrm{PbBr}_{4}$ excitonic absorptions with $n=1,2,3$ and 4 , respectively. ${ }^{[20,26]}$ These absorption characteristics imply that the $\mathrm{CsPbBr}_{3}+\mathrm{PEG}+\mathrm{PEABr}$ 


\section{WILEY-VCH}

perovskites are composed of mixed-dimensional perovskite phases including various quasi2D PEA $2\left(\mathrm{CsPbBr}_{3}\right)_{n-1} \mathrm{PbBr}_{4}$ structures with different exciton energies.

To understand the carrier dynamics in the $\mathrm{CspbBr}_{3}$ perovskite films, time-resolved PL decays were recorded at the wavelength of the PL emission peaks (Table S2). Figure $2 \mathrm{~d}$ plots the PL decay curves, which are fitted with a bi-exponential decay function consisting of a fast-decay component $\left(\tau_{1}\right)$ and a slow-decay component $\left(\tau_{2}\right) \cdot{ }^{[6]}$ Table S2 summarizes the characteristic decay times and the calculated average lifetimes $\left(\tau_{\text {avg }}\right)$. Pure $\mathrm{CsPbBr}_{3}$ perovskite exhibits the shortest PL lifetime of $\tau_{\text {avg }}=12.07$ ns. Notably, the addition of PEABr or PEG prolongs the PL lifetime of the perovskite films, suggesting that these two additives can passivate the defects at grain boundaries and suppress the defect-assisted non-radiative recombination. More importantly, the $\mathrm{CsPbBr}_{3}+\mathrm{PEG}+\mathrm{PEABr}$ perovskite has the longest lifetime of $\tau_{\text {avg }}=62.44 \mathrm{~ns}$ (Table S2). This means that PEABr and PEG have the synergetic effects on morphology control as well as defect passivation. As discussed above and shown in Figure S3, the addition of PEABr leads to the formation of mixed-dimensional perovskite phases, where energy funneling may occur from the larger $2 \mathrm{D}$ bandgap to the lower bulk $3 \mathrm{D}$ bandgap domains. To verify this viewpoint, the PL decay characteristics of the perovskite films at various emission wavelengths were further detected under the excitation of $370 \mathrm{~nm}$ laser pulses (Figure $\mathrm{S} 6$ ). As compared to the cases of pure $\mathrm{CsPbPb}_{3}$ and $\mathrm{CsPbPb}_{3}+\mathrm{PEG}$ films, the time-resolved PL decay curves (at $520 \mathrm{~nm}$ ) of the $\mathrm{CsPbPb}_{3}+\mathrm{PEABr}$ and $\mathrm{CsPbPb}_{3}+\mathrm{PEG}+\mathrm{PEABr}$ perovskite films exhibit clear rise times of 1-2 ns. However, such differences cannot be observed for the PL decays at other emission wavelengths (e.g., $420 \mathrm{~nm}$ and $460 \mathrm{~nm}$ ). This observation indicates that the filling time of excited states, of which the energy corresponds to the emission wavelength of $520 \mathrm{~nm}$, is quite slower in either the $\mathrm{CsPbPb}_{3}+\mathrm{PEABr}$ or the $\mathrm{CsPbPb}_{3}+\mathrm{PEG}+\mathrm{PEABr}$ perovskite film, suggesting that the excited state population in these two films primarily results from energy transfer happening within 1- 


\section{WILEY-VCH}

2 ns after laser pulse-induced direct excitation. Such observations are consistent with the previous works which demonstrated that efficient energy transfer from the larger 2D bandgap to the lower bulk 3D bandgap domains can enhance the radiative recombination. ${ }^{[1,12]}$

\subsection{Formation Mechanism}

Figure 3 illustrates the mechanisms of the morphology control over $\mathrm{CsPbBr}_{3}$ perovskite films with PEG and PEABr additives. During the volatilization of the DSMO solvent in the spin-coating process, the $\mathrm{CsPBBr}_{3}$ perovskite is continuously crystallized from the precursor solution with ionic interactions between metal cations and halogen anions. As the residual DMSO solvent dries slowly in the perovskite layer, the $\mathrm{Cs}_{\mathrm{PbBr}}$ perovskite usually grows into large grains, resulting in the incomplete surface coverage with numerous pinholes (Figure 3a). The $\mathrm{PEABr}$ additive can dramatically impede the continuous growth of $\mathrm{CsPbBr}_{3}$ perovskites by intercalating at the grain boundaries to restrain their bonding interaction (Figure $3 b$ ). During this process, partial replacement of $\mathrm{Cs}^{+}$ions with $\mathrm{PEA}^{+}$at the grain boundaries will take place and thus limit the grain growth of bulk 3D perovskite structure in one dimension, leading to the formation of $2 \mathrm{D}$ perovskite crystals in the formula of $\mathrm{PEA}_{2}\left(\mathrm{CsPbBr}_{3}\right)_{n-1} \mathrm{PbBr}_{4}$ with small grains. Similar to previous reports, ${ }^{[11,12]}$ such a mixed-dimensional perovskite film is composed of a mixture of various n-phases. The bandgap of mixed-dimensional $\mathrm{CsPbr}_{3}$ perovskite films will increase gradually with a continuous increase in PEABr content, leading to a blue-shift of the emission peak from $520 \mathrm{~nm}$ to $507 \mathrm{~nm}$ (Figure S3). Although the mixeddimensional perovskite structure has good stability, its surface roughness is relatively large that limits the device performance. PEG with good solubility and rheology is a kind of Lewis base that can bind with Lewis acid $\mathrm{Pb}^{2+} \cdot{ }^{[27]}$ Therefore, the addition of a small amount of PEG can limit the diffusion of the $\mathrm{CsPbBr}_{3}$ precursors through chemical interactions (Figure 3c). The improved morphology with low surface roughness and low pinhole densities in the perovskite films (Figure 2b) is thus realized since the PEG additive can promote the uniformity of the perovskite films as a matrix to fill the grain boundaries and passivate the 


\section{WILEY-VCH}

defects at the grain surface due to the defect healing effect. Accordingly, the combination of PEG and PEABr additives can induce the synergetic effect on the morphology control over mixed-dimensional $\mathrm{CsPBr}_{3}$ perovskite films with the reduced grain size and the passivation of defect density at the grain boundaries (Figure 3d). As compared with pure $\mathrm{CsPbBr}_{3}$ perovskite films, the suppression of current leakage and non-radiative recombination rate can be expected in the $\mathrm{CsPBr}_{3}+\mathrm{PEG}+\mathrm{PEABr}$ perovskite film because of the PL enhancement and the prolonged emission lifetime (Figure 2).

\subsection{Device Performance}

The PeLEDs are constructed using different $\mathrm{CsPbBr}_{3}$ perovskite films with the same device architecture of glass/ITO/PEDOT:PSS/ $\mathrm{CsPbBr}_{3}$ perovskite/TPBi/LiF/Al, where ITO is used as an anode, PEDOT:PSS as a hole-transport layer (HTL), $\mathrm{CsPbBr}_{3}$ perovskite as an emitting layer, 2,2',2”-(1,3,5-Benzinetriyl)- tris(1-phenyl-1-H-benzimidazole) (TPBi) as an electron-transport/hole-blocking layer (ETL/HBL), and $\mathrm{LiF} / \mathrm{Al}$ as a bilayer cathode (see the details in Experimental Section). Figure 4a shows the energy level diagram of the PeLED, in which the energy level positions of all function layers refer to the previous reports. ${ }^{[11,13,14]}$ It is expected that holes can be effectively confined for the exciton formation in the $\mathrm{CsPBr}_{3}$ perovskite films due to the deep highest occupied molecular orbital (HOMO) level of TPBi. The device characteristics of various PeLEDs are plotted in Figure $4 \mathrm{~b}-4 \mathrm{~g}$, and the detailed device performances are summarized in Table 1. The PeLED with pure $\mathrm{CsPbBr}_{3}$ exhibits a maximum luminance of $9,662 \mathrm{~cd} \mathrm{~m}^{-2}$, while the CE and EQE are limited to $2.5 \mathrm{~cd} \mathrm{~A}^{-1}$ and $0.76 \%$, respectively. Such poor performance may be ascribed to the large current leakage and high non-radiative recombination resulting from poor film morphology with numerous pinholes and defects at the grain boundaries. It is obvious in Figure $4 \mathrm{~b}$ that the addition of $\mathrm{PEG}$ or $\mathrm{PEABr}$ in the $\mathrm{CsPbBr}_{3}$ perovskite films induces lower current density as compared to that of pure $\mathrm{CsPbBr}_{3}$ under the same bias voltage, while a substantial increase in luminance and efficiency is achieved for PeLEDs using $\mathrm{CsPbBr}_{3}$ perovskite films with $\mathrm{PEG}$ or $\mathrm{PEABr}$ 


\section{WILEY-VCH}

additive (Figure 4b-4d). For example, the PeLED with $\mathrm{CsPbBr}_{3}+\mathrm{PEG}$ perovskite film exhibits the enhanced $\mathrm{CE}$ and EQE up to $5.05 \mathrm{~cd} \mathrm{~A}^{-1}$ and $1.39 \%$, respectively. As suggested by the SEM and PL decay results, such an efficiency improvement is attributed to the compact and pinhole-free morphology of the $\mathrm{Cs}_{\mathrm{PbBr}}+\mathrm{PEG}$ perovskite film and the reduced non-radiative recombination. The injection-current of PeLEDs with $\mathrm{CsPbBr}_{3}+\mathrm{PEABr}$ perovskite film is to some extent lower than that using pure $\mathrm{CsPbBr}_{3}$ or $\mathrm{CsPbBr}_{3}+\mathrm{PEG}$ perovskite film, which is mainly correlated to the limited charge transport properties of mixed-dimensional $\mathrm{CsPbBr}_{3}$ structure with small grain size and incomplete surface coverage. However, the remarkable enhancement in $\mathrm{CE}$ and $\mathrm{EQE}$ of the $\mathrm{CsPBr}_{3}+\mathrm{PEABr}$-based device indicates the increase in radiative recombination efficiency due to the presence of mixed-dimensional perovskite structure for more efficiently exciton generation as demonstrated in the PL measurement (Figure 2). Correspondingly, the combination of PEG and PEABr causes the synergetic effect on morphology control over $\mathrm{CsPBr}_{3}$ perovskite films with high surface coverage, which can suppress the current leakage and possibly improve the electron-hole balance for the increased radiative recombination efficiency. As a result, the PeLED using $\mathrm{CsPbBr}_{3}+\mathrm{PEG}+\mathrm{PEABr}$ perovskite film as the emitter outperforms the device with pure $\mathrm{CsPbBr}_{3}$ by an order of magnitude in both luminance and efficiency. For instance, a maximum luminance of 45,990 $\mathrm{cd} \mathrm{m}^{-2}$ is obtained for the $\mathrm{CsPbBr} 3+\mathrm{PEG}+\mathrm{PEABr}-$ based PeLED along with a maximum $\mathrm{CE}$ of $37.14 \mathrm{~cd} \mathrm{~A}^{-1}$ and a maximum EQE of $13.14 \%$, respectively, which are 17 times that of the device with pure $\mathrm{CsPbBr}_{3}$ (Table 1). More remarkably, the device shows small efficiency rolloff at high luminance. The EQE of the $\mathrm{CsPbBr}_{3}+\mathrm{PEG}+\mathrm{PEABr}$-based device remains at a high value of $9.3 \%$ along with a $\mathrm{CE}$ of $31.2 \mathrm{~cd} \mathrm{~A}^{-1}$ even at a high luminance of $10,000 \mathrm{~cd} \mathrm{~m}^{-2}$. In addition, the EQE histogram displayed in Figure 4e reveals high reproducibility of the $\mathrm{CsPbBr}_{3}+\mathrm{PEG}+\mathrm{PEABr}$-based PeLED. The variation of the device performance of PeLEDs with different perovskite films can be correlated to the film quality, which is consistent with the comparison of morphological and optical properties among $\mathrm{CsPbBr}_{3}$ perovskites with 


\section{WILEY-VCH}

different PEG and PEABr contents (Figures S4-S5 and Table S3). The normalized electroluminescence (EL) spectra of the PeLEDs in Figure 4f exhibit single green emission centered at the same wavelengths as the PL peaks (Table 1), and the EL spectra are stable under various bias voltages (Figure S7). Stable and clean EL emission can be obtained under continuous operation of the devices (inset in Figure 4f). In addition, the EL spectra show excellent color purity (85\%) in the green region with a narrow FWHM of $\sim 18 \mathrm{~nm}$. The corresponding Commission Internationale de I'Eclairage (CIE) chromaticity coordinates (x, y) of the $\mathrm{CsPbBr}_{3}+\mathrm{PEG}+\mathrm{PEABr}-$ based PeLEDs are $(0.092,0.753)$ (Figure 4g). These results demonstrate that the performance enhancement of $\mathrm{CsPbBr}_{3}$ PeLEDs is primarily attributed to the synergetic interplay of PEG and PEABr additives on the morphology optimization which results in a smooth and pinhole-free mixed-dimensional perovskite film, enabling the drastic boost in radiative recombination efficiency due to the efficient energy funneling and spatial carrier confinement in the lowest bandgap 3D phase.

For bendable display and curved lighting applications with PeLEDs, it is highly desirable to realize the flexible device architecture on plastic substrates. However, the efficiency values of flexible PeLEDs reported so far lag behind those of the counterparts on rigid glass substrates. ${ }^{[28-31]}$ To validate the effectiveness of the method in this work, flexible PeLEDs using the $\mathrm{CsPbBr}_{3}+\mathrm{PEG}+\mathrm{PEABr}$ perovskite film were further fabricated on polyethylene terephthalate (PET) substrates, in which Ag nanowires (AgNWs) was used as the transparent electrode to replace the conventional ITO ${ }^{[32-34]}$ As shown in Figure 5a, the AgNWs with a diameter of $\sim 40 \mathrm{~nm}$ and a length of $20-30 \mu \mathrm{m}$ were spin-cast on PET substrates to form a transparent electrode, which possess an optical transmittance of $>80 \%$ in the visible wavelength region and a sheet resistance of $<25 \Omega \mathrm{sq}^{-1}$. The root-mean-square (RMS) roughness of the AgNWs electrode was determined to be $\sim 20 \mathrm{~nm}$ (Figure S8a). To smooth the electrode surface, a thicker PEDOT:PSS layer with a thickness of $45 \mathrm{~nm}$ was spin-coated on the AgNWs-coated PET substrate, resulting in a RMS roughness of $\sim 6 \mathrm{~nm}$. The morphology 


\section{WILEY-VCH}

of the $\mathrm{CsPbBr}_{3}+\mathrm{PEG}+\mathrm{PEABr}$ perovskite film grown on flexible PET substrates was measured (Figure 5, Figure S8b), which is almost identical to that on glass substrates. It is noteworthy that flexible PeLED with $\mathrm{CsPbBr}_{3}+\mathrm{PEG}+\mathrm{PEABr}$ perovskite film exhibits a high performance with a maximum EQE of $10.1 \%$ and a maximum $\mathrm{CE}$ of $31.0 \mathrm{~cd} \mathrm{~A}^{-1}$ (Figure 5c), respectively, which are comparable to the glass-based counterparts. These results indicate that the proposed formation method of the $\mathrm{CsPbBr}_{3}+\mathrm{PEG}+\mathrm{PEABr}$ perovskite film can be effectively used on plastic substrates to obtain high-performance flexible devices.

\section{Conclusions}

In summary, bright all-inorganic PeLEDs with high EQEs have been demonstrated by synergetic morphology control over $\mathrm{CsPBr}_{3}$ perovskite films with the combination of $\mathrm{PEABr}$ and PEG additives. The PEABr additive enables the formation of mixed-dimensional $\mathrm{CsPbBr}_{3}$ perovskites with small grain size that feature efficient energy funneling, whereas the dielectric PEG additive promotes the morphological uniformity of the perovskite films as a matrix to fill the grain boundaries and passivate the grain surface with reduced defect density. The improved morphology with pinhole-free surface coverage can significantly suppress the current leakage and non-radiative recombination in the resulting perovskite film. Substantial increase in both brightness and efficiency has been achieved in all-inorganic green PeLEDs, among which the best device yields a current efficiency of $37.14 \mathrm{~cd} \mathrm{~A}^{-1}$, an EQE of $13.14 \%$, a maximum luminance of 45,990 $\mathrm{cd} \mathrm{m}^{-2}$ and high color purity. To the best of our knowledge, the maximum EQE of our devices is among the highest efficiencies for all-inorganic $\mathrm{CsPbr}_{3}-$ based PeLEDs reported so far (Table S4). Furthermore, efficient flexible PeLEDs with stateof-the-art performance were realized on plastic substrates, showing a CE of $31.0 \mathrm{~cd} \mathrm{~A}^{-1}$ and an EQE of $10.1 \%$. We anticipate that this approach may provide a facile route to improving the halide perovskite-based devices with desirable morphological, optical and electrical properties.

\section{Experimental Section}




\section{WILEY-VCH}

Perovskite Preparation: $\mathrm{CsBr}$ and $\mathrm{PbBr}_{2}$ were purchased from TCI (99.0\%). PEG (average Mv 600000), dimethyl sulfoxide (DMSO) (99.7\%) and LiF (99.99\%) were purchased from MACKLIN. TPBi (99.0\%) was purchased from Nichem. PEABr (99.5\%) was purchased from Xi'an Polymer. PEDOT:PSS (Clevios P VP Al 4083) was purchased from Heraeus. All the materials were used directly without any treatment. The synthetic procedure of the $\mathrm{CsPbBr}_{3}$ perovskite films with PEG and PEABr additives is shown in Figure 1a. Firstly, the $\mathrm{CsPbBr}_{3}$ precursor solution was prepared by simultaneously dissolving $\mathrm{CsBr}\left(60 \mathrm{mg} \mathrm{mL}^{-1}\right), \mathrm{PbBr}_{2}(70$ $\left.\mathrm{mg} \mathrm{mL}^{-1}\right)$, and PEABr $\left(13 \mathrm{mg} \mathrm{mL}^{-1}\right)$ in DMSO. Then, the PEG $\left(10 \mathrm{mg} \mathrm{mL}^{-1}\right)$ in DMSO was mixed with the $\mathrm{CsPBr}_{3}$ solution to obtain a transparent precursor solution, which was stirred for a few hours at room temperature. Finally, the $\mathrm{CsPbBr}_{3}$ perovskite films were obtained by spin-coating the precursor solution at $3000 \mathrm{rpm}$ for $60 \mathrm{~s}$ in a nitrogen-filled glovebox, and subsequently annealed on a hot plate at $70{ }^{\circ} \mathrm{C}$ for $5 \mathrm{~min}$ to remove the DMSO residual.

Device Fabrication: The PeLEDs were constructed with a structure of glass/ITO/PEDOT:PSS/CsPbBr 3 perovskite/TPBi/LiF/Al, in which ITO with a sheet resistance of $\sim 10 \Omega \mathrm{sq}^{-1}$ was used as anode, PEDOT:PSS as hole-transport layer, $\mathrm{CsPbBr}_{3}$ perovskite layer as the green emitter, TPBi as electron-transport layer, and LiF/Al as a bilayer cathode, respectively. ITO-coated glass substrates were cleaned by ultrasonication in detergent, acetone, ethanol, and deionized water for $30 \mathrm{~min}$ in sequence, and subsequently dried in an oven $\left(110^{\circ} \mathrm{C}\right)$. Prior to the layer deposition, ITO-coated glass substrates were treated by UV ozone for $15 \mathrm{~min}$. The filtered PEDOT:PSS solution with $0.45 \mu \mathrm{m}$ poly(tetrafluoroethylene) syringe filters was spin-coated on ITO glass substrates at $4000 \mathrm{rpm}$ for $40 \mathrm{~s}$, and annealed at $140{ }^{\circ} \mathrm{C}$ for $15 \mathrm{~min}$ in ambient air to form a $40 \mathrm{~nm}$-thick film. The samples were then transferred into a $\mathrm{N}_{2}$-filled glovebox and spin-coated with various $\mathrm{CsPbr}_{3}$ precursor solutions. Finally, the samples with $\mathrm{CsPbBr}_{3}$ perovskite films were transferred from the $\mathrm{N}_{2}$-filled glovebox into an interconnected high-vacuum deposition system (base pressure 


\section{WILEY-VCH}

$\approx 5 \times 10^{-7}$ Torr $)$ for thermal evaporation of TPBi $(40 \mathrm{~nm})$ and $\mathrm{LiF}(1 \mathrm{~nm}) / \mathrm{Al}(100 \mathrm{~nm})$ through shadow masks to complete the device. The deposition rate and film thickness were monitored with a quartz crystal oscillator. The effective area of PeLEDs was determined to be $10 \mathrm{~mm}^{2}$ with the overlap between ITO and Al electrodes. The encapsulation of PeLEDs was carried out with glass lid and epoxy resin. To get consistent results, devices with different perovskite films were usually fabricated in the same batch.

Characterization: The morphologies of $\mathrm{CsPbBr} 3$ perovskite films were characterized by fieldemission scanning electron microscopy (SEM) (Zeiss Supra 55). XRD patterns were recorded with a Rigaku $\mathrm{D} /$ MaxrB diffraction with $\mathrm{Cu} \mathrm{K} \alpha$ radiation to characterized the crystal structures of the perovskite films. The ultraviolet-visible absorption spectra were measured with a UV/vis/near-IR spectrophotometer (Perkin Elmer Lambda 750). Steady-state PL spectra and time-resolved PL decays were collected with FluoroMax-4 fluorescence spectrometer (Horiba Jobin Yvon) and Horib-FM-2015 spectrometer, respectively. Current density-voltage-luminance (I-V-L) characteristics and electroluminescence (EL) spectra of PeLEDs were measured simultaneously with a computer-controlled programmable power source (Keithley model 2400) and a luminance meter/spectrometer (PhotoResearch PR655). The EQEs were calculated by taking a full account of the measured I-V-L characteristics and EL spectra of PeLEDs with a Lambertian profile. All the measurements were carried out under ambient conditions at room temperature.

\section{Supporting Information}

Supporting Information is available from the Wiley Online Library or from the author.

\section{Acknowledgements}

L.-P.C. and J.-S.H. contributed equally to this work. This work was financially supported by the National Natural Science Foundation of China (Nos. 61520106012, 61722404, 61522505), 


\section{WILEY-VCH}

the National Key R\&D Program of China (Nos. 2016YFB0401002, 2016YFB0400700), Collaborative Innovation Center of Suzhou Nano Science and Technology, and the project of the Priority Academic Program Development (PAPD) of Jiangsu Higher Education Institutions. X.-K.L. is a Marie Skłodowska-Curie Fellow. F.G. thanks the support from ERC Starting Grant (No. 717026).

Received: ((will be filled in by the editorial staff))

Revised: ((will be filled in by the editorial staff)) Published online: ((will be filled in by the editorial staff))

\section{References}

[1] Z.-K. Tan, R. S. Moghaddam, M. L. Lai, P. Docampo, R. Higler, F. Deschler, M. Price, A. Sadhanala, L. M. Pazos, D. Credgington, F. Hanusch, T. Bein, H. J. Snaith, R. H. Friend, Nat. Nanotechnol. 2014, 9, 687-692.

[2] L. Protesescu, S. Yakunin, M. I. Bodnarchuk, F. Krieg, R. Caputo, C. H. Hendon, R. X. Yang, A. Walsh, M. V. Kovalenko, Nano Lett. 2015, 15, 3692-3696.

[3] G. Xing, N. Mathews, S.S. Lim, N. Yantara, X. Liu, D. Sabba, M. Grätzel, S. Mhaisalkar, T. C. Sum, Nat. Mater. 2014, 13, 476-480.

[4] J. Song, J. Li, X. Li, L. Xu, Y. Dong, H. Zeng, Adv. Mater. 2015, 27, 7162-7167.

[5] M. Kulbak, D. Cahen, G. Hodes, J. Phys. Chem. Lett. 2015, 6, 2452-2456.

[6] H. Cho, S.-H. Jeong, M.-H. Park, Y.-H. Kim, C. Wolf, C.-L. Lee, J. H. Heo, A. Sadhanala, N. Myoung, S. Yoo, S. H. Im, R. H. Friend, T.-W. Lee, Science 2015, 350, 1222-1225.

[7] X. Li, Y. Wu, S. Zhang, B. Cai, Y. Gu, J. Song, H. Zeng, Adv. Funct. Mater. 2016, 26, $2435-2445$.

[8] X. Zhang, B. Xu, J. Zhang, Y. Gao, Y. Zheng, K. Wang, X. W. Sun, Adv. Funct. Mater. 2016, 26, 4595-4600. 


\section{WILEY-VCH}

[9] Y. Ling, Z. Yuan, Y. Tian, X. Wang, J. C. Wang, Y. Xin, K. Hanson, B. Ma, H. Gao, Adv. Mater. 2016, 28, 305-311.

[10] J. Li, L. Xu, T. Wang, J. Song, J. Chen, J. Xue, Y. Dong, B. Cai, Q. Shan, B. Han, H. Zeng, Adv. Mater. 2017, 29, 1603885.

[11] X. L. Yang, X. W. Zhang, J. X. Deng, Z. M. Chu, Q. Jiang, J. H. Meng, P. Y. Wang, L. Q. Zhang, Z. G. Yin, J. B. You, Nat. Commun. 2018, 9, 570.

[12] N. Wang, L. Cheng, R. Ge, S. Zhang, Y. Miao, W. Zou, C. Yi, Y. Sun, Y. Cao, R. Yang, Y. Wei, Q. Guo, Y. Ke, M. Yul Y. Jin, Y. Liu, Q. Ding, D. Di, L. Yang, G. Xing, H. Tian, C. Jin, F. Gao, R. H. Friend, J. Wang, W. Huang, Nat. Photon. 2016, 10, 699-704.

[13] Z. G. Xiao, R. A. Kerner, L. F. Zhao, N. L. Tran, K. M. Lee, T.-W. Koh, G. D. Scholes, B. P. Rand, Nat. Photon. 2017, 11, 108-115.

[14] Y. Ling, Y. Tian, X. Wang, J. C. Wang J. M. Knox, F. Perez-Orive, Y. Du, L. Tan, K. Hanson, B. Ma, H. Gao, Adv. Mater. 2016, 28, 8983-8989.

[15] G. Li, F. W. R. Rivarola, N. J. L. K. Davis, S. Bai, T. C. Jellicoe, F. de la Peña, S. Hou, C. Ducati, F. Gao, R. H. Friend, N. C. Greenham, Z.-K. Tan, Adv. Mater. 2016, 28, 35283534.

[16] X. Zhang, H. Lin, H. Huang, C. Reckmeier, Y. Zhang, W. C. H. Choy, A. L. Rogach, Nano Lett. 2016, 16, 1415-1420.

[17] R. E. Beal, D. J. Slotcavage, T. Leijtens, A. R. Bowring, R. A. Belisle, W. H. Nguyen, G. F. Burkhard, E. T. Hoke, M. D. McGehee, J. Phys. Chem. Lett. 2016, 7, 746-751.

[18] N. Yantara, S. Bhaumik, F. Yan, D. Sabba, H. A. Dewi, N. Mathews, P. P. Boix, H. V. Demir, S. Mhaisalkar, J. Phys. Chem. Lett. 2015, 6, 4360-4364.

[19] L. Zhang, X. Yang, Q. Jiang, P. Wang, Z. Yin, X. Zhang, H. Tan, Y. Yang, M. Wei, B. R. Sutherland, E. H. Sargent, J. You, Nat. Commun. 2017, 8, 15640.

[20] Y. F. Ng, S. A. Kulkarni, S. Parida, N. F. Jamaludin, N. Yantara, A. Bruno, C. Soci, S. Mhaisalkar, N. Mathews, Chem. Commun. 2017, 53, 12004-12007. 


\section{WILEY-VCH}

[21] M. Yuan, L. N. Quan, R. Comin, G. Walters, R. Sabatini, O. Voznyy, S. Hoogland, Y. Zhao, E. M. Beauregard, P. Kanjanaboos, Z. Lu, D. H. Kim and E. H. Sargent, Nat. Nano. 2016, 11, 872-877.

[22] J. Byun, H. Cho, C. Wolf, M. Jang, A. Sadhanala, R. H. Friend, H. Yang and T.-W. Lee, Adv. Mater. 2016, 28, 7515-7520.

[23] L. N. Quan, Y. Zhao, F. P. García de Arquer, R. Sabatini, G. Walters, O. Voznyy, R. Comin, Y. Li, J. Z. Fan, H. Tan, J. Pan, M. Yuan, O. M. Bakr, Z. Lu, D. H. Kim, E. H. Sargent, Nano Lett. 2017, 17, 3701-3709.

[24] M. Kulbak, D. Cahen, G. Hodes, J. Phys. Chem. Lett. 2015, 6, 2452-2456.

[25] D. Liang, Y. Peng, Y. Fu, M. J. Shearer, J. Zhang, J. Zhai, Y. Zhang, R. J. Hamers, T. L. Andrew, S. Jin, ACS Nano 2016, 10, 6897-6904.

[26] L. N. Quan, M. Yuan, R. Comin, O. Voznyy, E. M. Beauregard, S. Hoogland, A. Buin, A. R. Kirmani, K. Zhao, A. Amassian, D. H. Kim, E. H. Sargent, J. Am. Chem. Soc. 2016, $138,2649-2655$.

[27] L. Song, X. Y. Guo, Y. S. Hu, Y. Lv, J. Lin, Z. Q. Liu, Y. Fan, X. Y. Liu, J. Phys. Chem. Lett. 2017, 8, 4148-4154.

[28] S. G. R. Bade, J. Li, X. Shan, Y. Ling, Y. Tian, T. Dilbeck, T. Besara, T. Geske, H. Gao, B. Ma, K. Hanson, T. Siegrist, C. Xu, Z. Yu, ACS Nano 2016, 10, 1795-1801.

[29] S. G. R. Bade, X. Shan, P. T. Hoang, J. Li, T. Geske, L. Cai, Q. Pei, C. Wang, Z. Yu, Adv. Mater. 2017, 29, 1607053.

[30] H.-K. Seo, H. Kim, J. Lee, M.-H. Park, S.-H. Jeong, Y.-H. Kim, S.-J. Kwon, T.-H. Han, S. Yoo, T.-W. Lee, Adv. Mater. 2017, 29, 1605587.

[31] G. Li, Z.-K. Tan, D. Di, M. L. Lai, L. Jiang, J. H.-W. Lim, R. H. Friend, N. C. Greenham, Nano Lett. 2015, 15, 2640-2644.

[32] J. Liang, L. Li, X. Niu, Z. Yu, Q. Pei, Nat. Photon. 2013, 7, 817-824. 


\section{WILEY-VCH}

[33] T. Sannicolo, M. Lagrange, A. Cabos, C. Celle, J. P. Simonato, D. Bellet, Small 2016, 12, 6052-6075.

[34] D. Kim, Y. Fu, S. Kim, W. Lee, K.-H. Lee, H. K. Chung, H.-J. Lee, H. Yang, H. Chae, ACS Nano 2017, 11, 1982-1990. 


\section{WILEY-VCH}

a

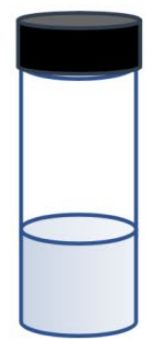

$\mathrm{CsPbBr}+\mathrm{PEABr}$ in DMSO

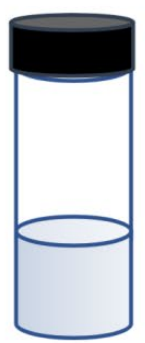

PEG in DMSO

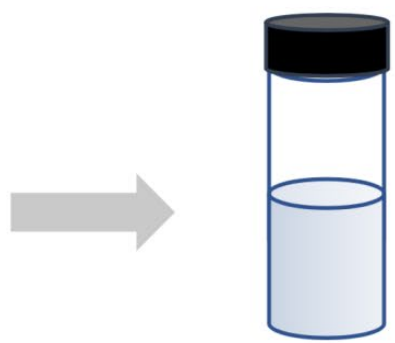

Precursor solution

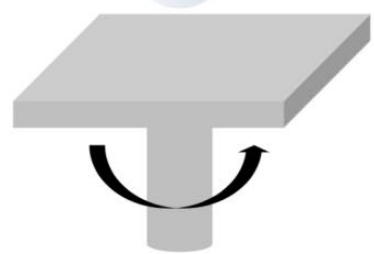

Spin coating

b

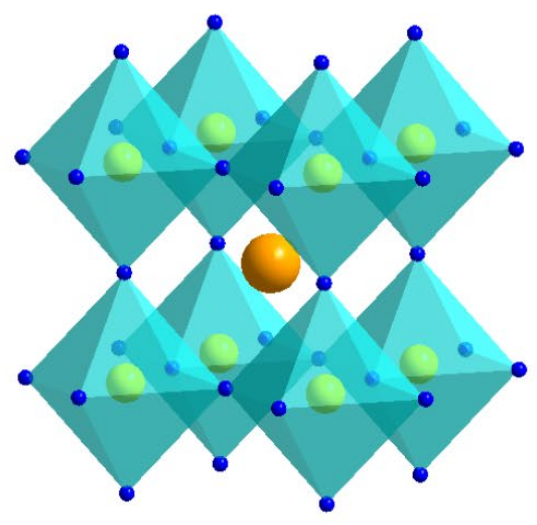

$\mathrm{CsPbBr}_{3}$

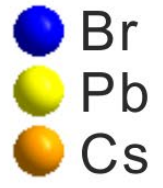

Cs

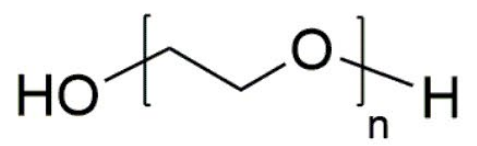

PEG

Figure 1. Synthesis of $\mathrm{CsPBrr}_{3}$-based perovskite thin films. (a) Schematic of the facile solution-based fabrication procedure of the perovskite film with PEABr and PEG additives. (b) Chemical structures of $\mathrm{CsPbBr}_{3}, \mathrm{PEABr}$ and PEG. 


\section{WILEY-VCH}
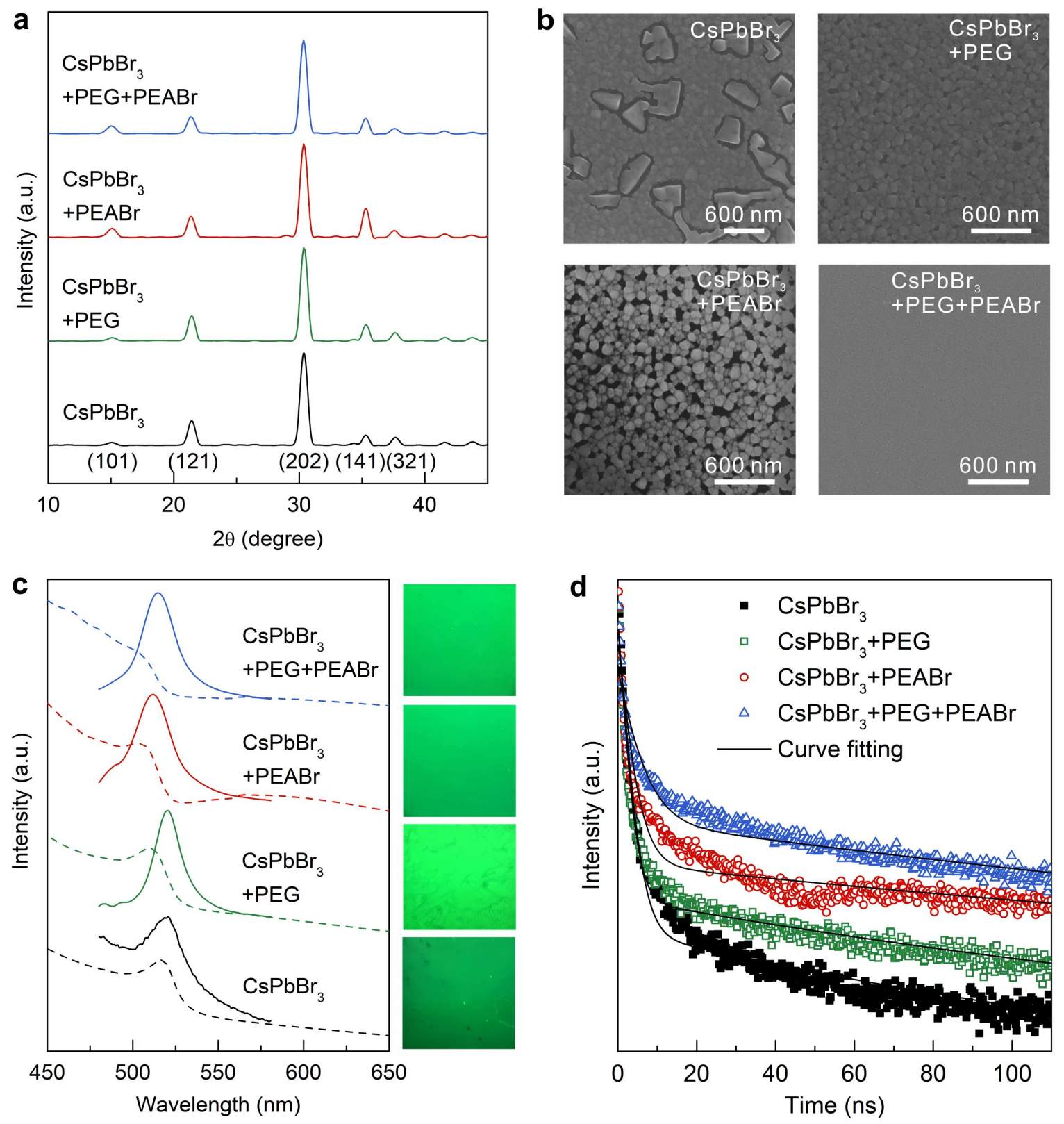

Figure 2. Properties of $\mathrm{CsPbBr}$. perovskite films. (a) XRD patterns of various $\mathrm{CsPbBr}_{3}$ perovskite films on PEDOT:PSS-coated ITO-glass substrates. (b) SEM images of $\mathrm{CsPbBr}_{3}$ perovskite films with different additives. (c) Absorption (dashed line) and PL (solid line) spectra of $\mathrm{CsPbBr}_{3}$ perovskite thin films on quartz substrates. Inserts: PL images of the $\mathrm{CsPbBr}_{3}$ perovskite films under ultraviolet lamp excitation (Hg lamp, $365 \mathrm{~nm}$ ). (d) Timeresolved $\mathrm{PL}$ decay curves of $\mathrm{CsPbr}_{3}$ perovskite films measured under the excitation of 370 nm. 


\section{WILEY-VCH}

a

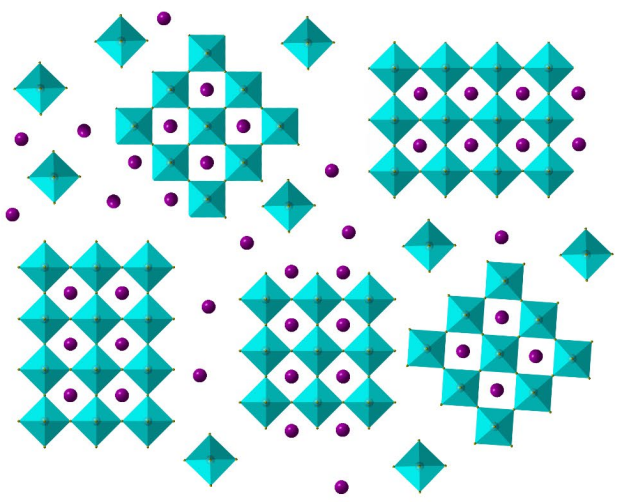

$\mathrm{CsPbBr}_{3}$

C

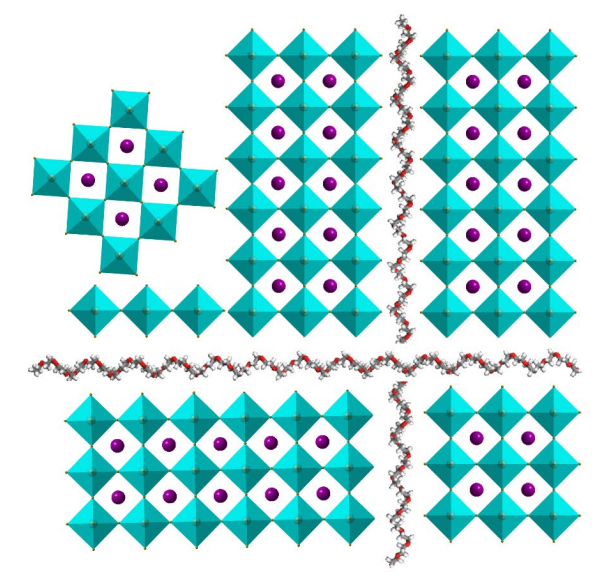

$\mathrm{CsPbBr}_{3}+\mathrm{PEG}$

C Cs
$P \mathrm{PbBr}_{6}$-octahedron b
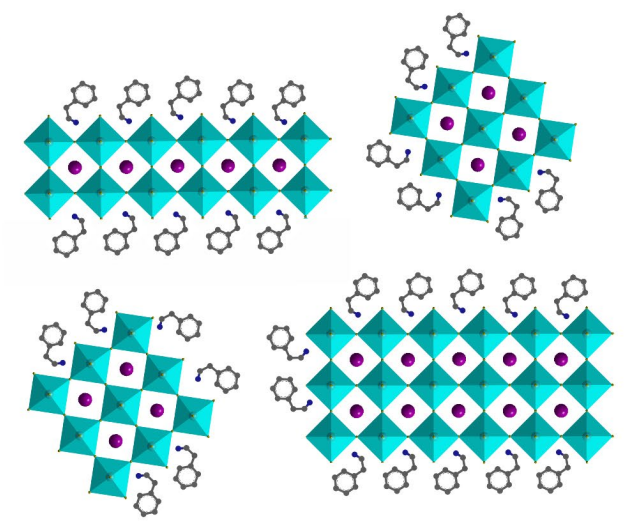

$\mathrm{CsPbBr}_{3}+\mathrm{PEABr}$

d

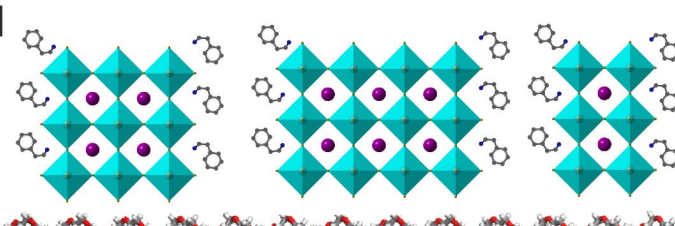

+

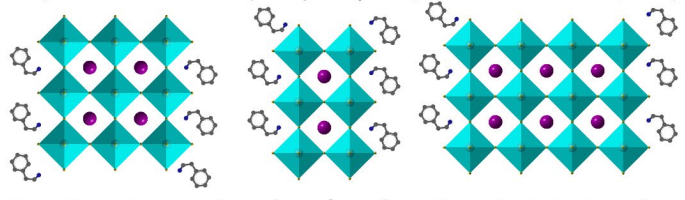

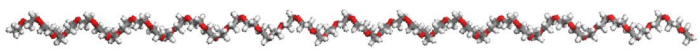
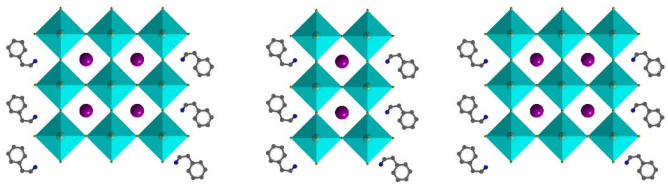

$\mathrm{CsPbBr}{ }_{3}+\mathrm{PEG}+\mathrm{PEABr}$

SET PEG

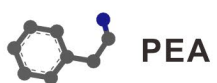

Figure 3. Schematic mechanism of the $\mathrm{CsPbBr}_{3}$ morphology control with the additives. (a)

Pure $\mathrm{CsPbBr}_{3}$ without any additive. (b) $\mathrm{CsPbBr}_{3}$ with $\mathrm{PEABr}$. (c) $\mathrm{CsPbBr}_{3}$ with PEG. (d) $\mathrm{CsPbBr}_{3}$ with both PEG and PEABr. 


\section{WILEY-VCH}

a
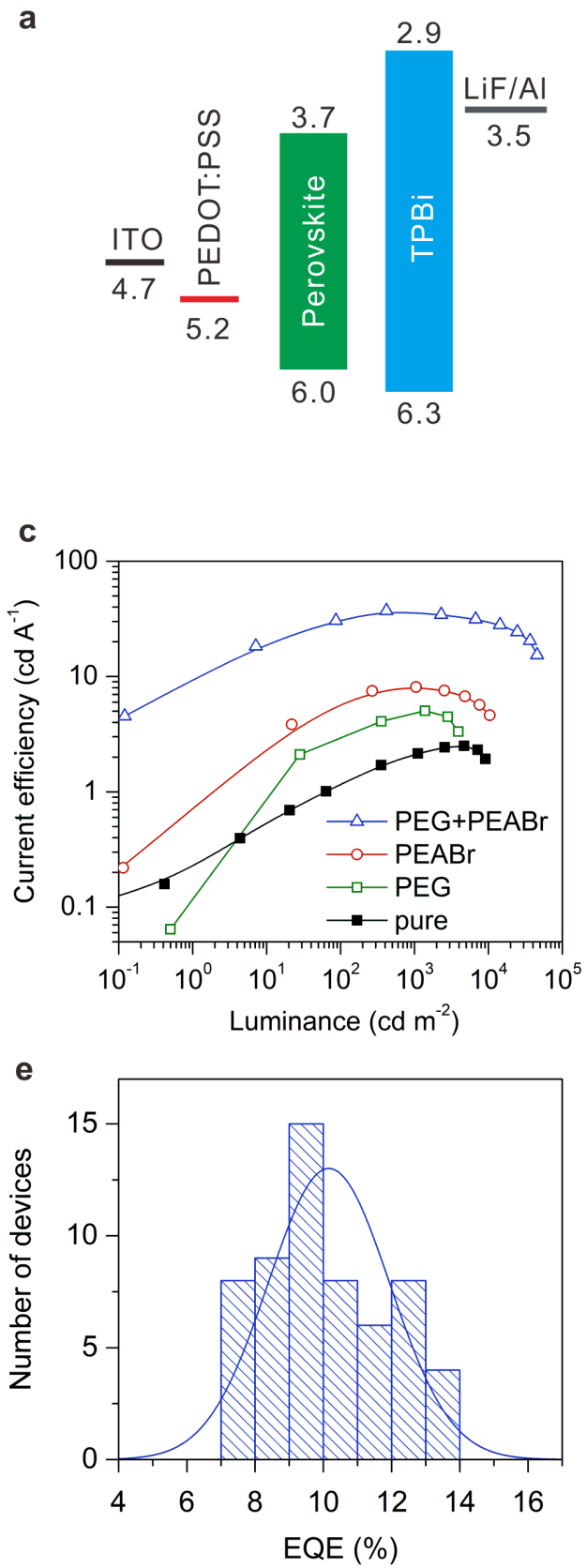

b

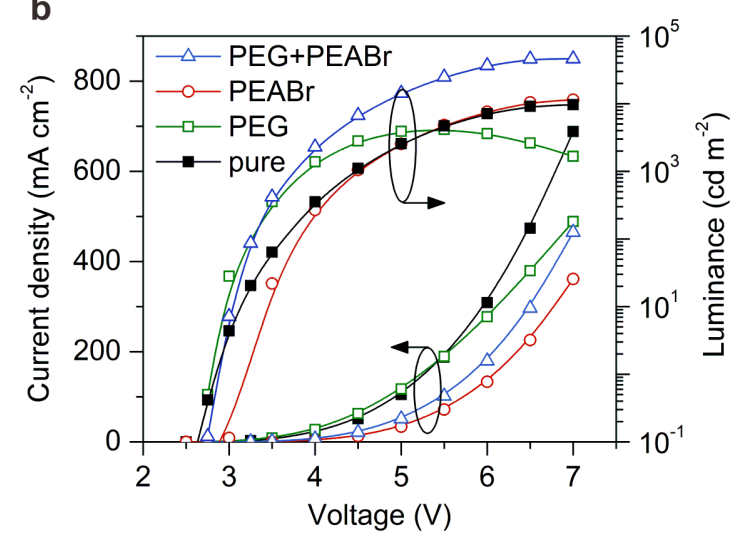

d

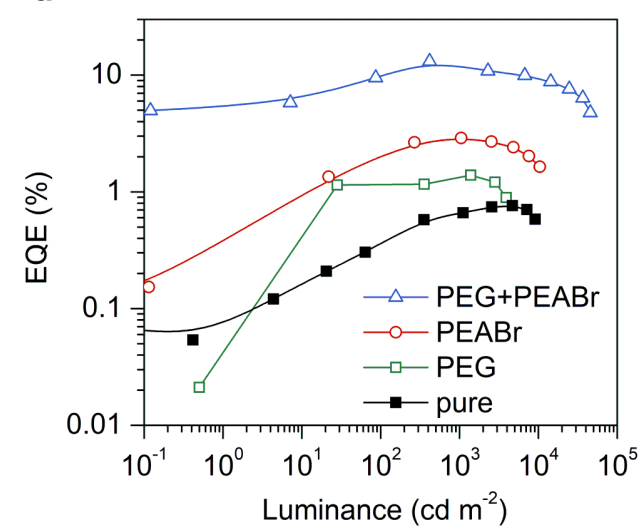

f

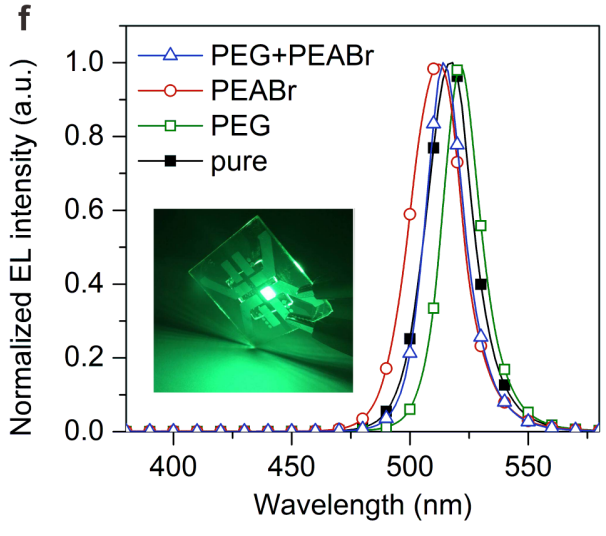

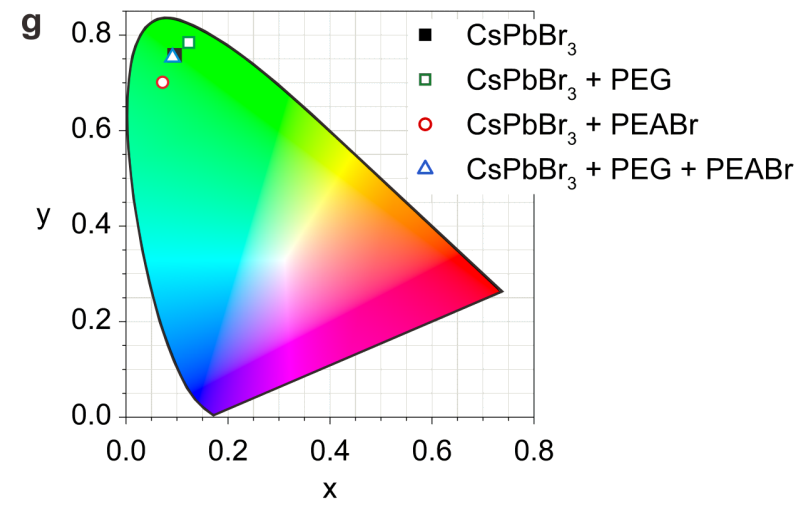

Figure 4. Device structure and performance of $\mathrm{CsPbBr}_{3}$ PeLEDs. (a) Energy level diagram of a $\mathrm{CsPbBr}_{3} \mathrm{PeLED}$ consisting of ITO/PEDOT:PSS (40 nm)/CsPbBr 3 (50 nm)/TPBi (40 


\section{WILEY-VCH}

$\mathrm{nm}) / \mathrm{LiF}(1 \mathrm{~nm}) / \mathrm{Al}(100 \mathrm{~nm})$ (unit: eV). (b) Current density-voltage (I-V) and luminancevoltage (L-V) characteristics. (c) Current efficiency as a function of luminance. (d) EQE as a function of luminance. (e) Histogram of peak EQEs measured from the $\mathrm{CsPbBr}_{3}+\mathrm{PEG}+\mathrm{PEABr}-$ based PeLEDs, which shows an average EQE of $10.3 \%$ with a relative standard deviation of $17.5 \%$. (f) EL spectra of various devices working at $4.5 \mathrm{~V}$. Inset: photograph of a device with both PEABr and PEG. (g) The Commission Internationale de I'Eclairage (CIE) coordinates of various $\mathrm{CsPbBr}_{3}$ PeLEDs. 


\section{WILEY-VCH}

a

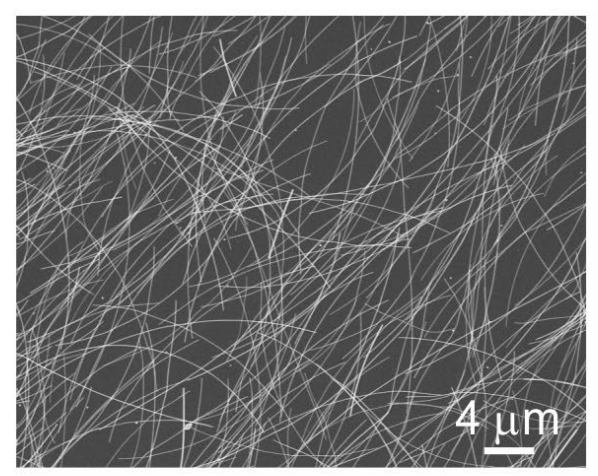

b

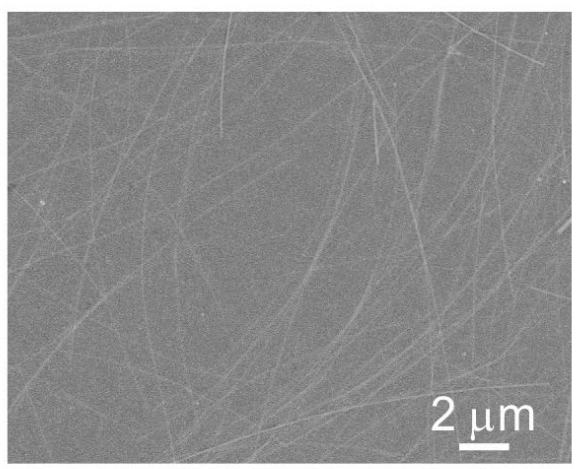

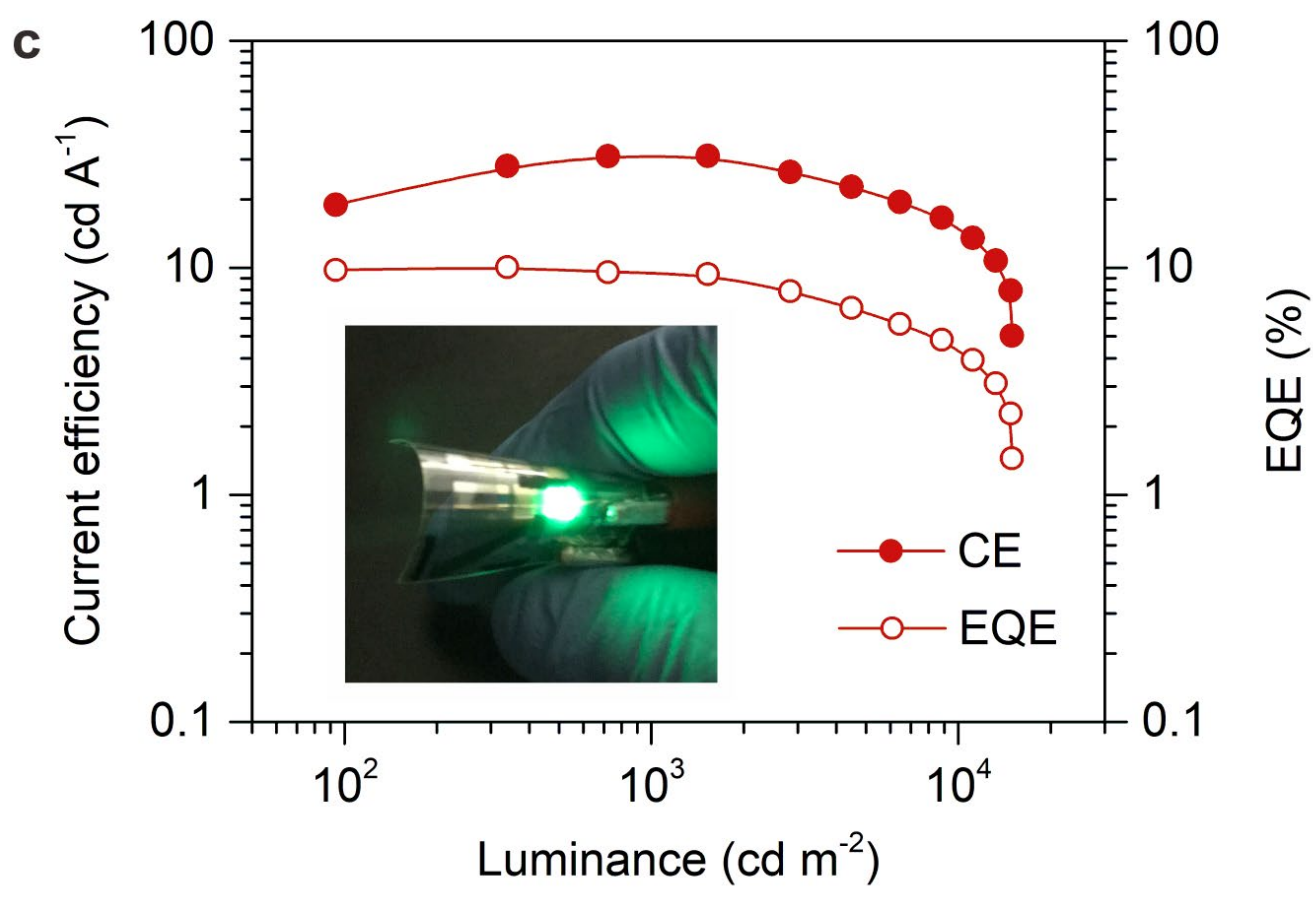

Figure 5. Performance of flexible PeLED on AgNWs-coated PET substrate. (a,b) SEM images of (a) AgNWs electrode on PET substrate and (b) $\mathrm{CsPbr}_{3}+\mathrm{PEG}+\mathrm{PEABr}$ perovskite film on PEDOT:PSS/AgNWs/PETe. (c) CE and EQE as a function of luminance. Inset: photograph of a flexible device in operation. 


\section{WILEY-VCH}

Table 1. Performance of $\mathrm{CsPbBr}_{3}$ PeLEDs without and with PEG and PEABr additives.

\begin{tabular}{cccccc}
\hline $\begin{array}{c}\text { CsPbBr } \\
\text { emitter }\end{array}$ & $\begin{array}{c}\text { PL/EL peak } \\
{[\mathrm{nm}]}\end{array}$ & $\begin{array}{c}\text { Max. L } \\
{\left[\mathrm{cd} \mathrm{m}^{-2}\right]}\end{array}$ & $\begin{array}{c}\text { EQE } \\
{[\%]}\end{array}$ & $\begin{array}{c}\text { CE } \\
{\left[\mathrm{cd} \mathrm{A}{ }^{-1}\right]}\end{array}$ & $\begin{array}{c}\text { CIE } \\
{[\mathrm{x}, \mathrm{y}]}\end{array}$ \\
\hline pure & $520 / 518$ & 9662 & 0.76 & 2.50 & {$[0.096,0.750]$} \\
PEG & $520 / 520$ & 4161 & 1.39 & 5.05 & {$[0.124,0.783]$} \\
PEABr & $512 / 512$ & 11430 & 2.89 & 8.09 & {$[0.072,0.700]$} \\
PEG+PEABr & $514 / 514$ & 45990 & 13.14 & 37.14 & {$[0.092,0.753]$} \\
\hline
\end{tabular}

